\title{
Ribociclib in Breast Cancer Brain Metastases: A Case Report
}

\author{
Isabel Radke ${ }^{a}$ Marie-Kristin von Wahlde ${ }^{a}$ Christoph Schülke ${ }^{b} \quad$ Joke Tio ${ }^{a}$ \\ ${ }^{a}$ Department of Gynecology and Obstetrics, University Hospital of Münster, Münster, Germany; ${ }^{b}$ Department of \\ Radiology, University Hospital of Münster, Münster, Germany
}

\section{Established Facts}

- The incidence of breast cancer brain metastases increases. But evidence-based therapeutic options of systemic treatment are limited because these patients are often excluded from clinical trials.

- Endocrine combination therapies with CDK4/6 inhibitors are widely used in first- and further-line treatment of hormone receptor-positive, HER2-negative breast cancer. Little is known about the effectiveness of these treatments in patients with breast cancer brain metastases.

\section{Novel Insights}

- Endocrine combination therapy with ribociclib in breast cancer brain metastases led to a fast and durable response even without surgical and/or radiation treatment.

- Endocrine combination therapy with ribociclib is a therapeutic option for patients with breast cancer brain metastases.

\section{Keywords}

Breast cancer brain metastases - Endocrine therapy · CDK4/6 inhibitor $\cdot$ Ribociclib

\begin{abstract}
Introduction: Breast cancer is the most common cancer in women. It frequently metastasizes to the lung, liver, and bones. Due to the improvement of therapeutic strategies and therefore longer patient survival, brain metastases have become more frequent. However, evidence-based therapeutic options of systemic treatment are limited because patients with breast cancer brain metastases are often excluded from clinical trials. Case Presentation: Here, we show a patient with brain and orbital metastases from a hormone receptor-positive, Her2neu-negative breast cancer that led to one-sided blindness. She was treated with a combination
\end{abstract}

therapy of the CDK4/6 inhibitor ribociclib and the aromatase inhibitor anastrozole and showed a fast and durable response for 9 months with good tolerability of the treatment. Conclusion: Systemic treatment with a CDK4/6 inhibitor and endocrine therapy can be considered in breast cancer brain metastases.

(c) 2019 S. Karger AG, Basel

\section{Introduction}

Breast cancer is the most common cancer in women and the leading cause of cancer-related death [1]. It frequently causes metastases in the lung, liver, and bones [2]. In addition, the incidence of brain metastases is increasing as better systemic therapies lead to improved disease control and longer patient survival [3]. Furthermore, di- 
Table 1. Size of the parenchymal brain metastases in $\mathrm{mm}$ at baseline and at the follow-up 3.5 months after initiating treatment with ribociclib and anastrozole

\begin{tabular}{lcrll}
\hline & Frontal & Occipital & Parietal 1 & Parietal 2 \\
\hline Baseline & $9.3 \times 7.4$ & $11.7 \times 8.5$ & $19.1 \times 10.9$ & $9.7 \times 9.2$ \\
Follow-up & $9.0 \times 7.4$ & $8.7 \times 6.0$ & $15.0 \times 6.8$ & $8.7 \times 5.2$ \\
\hline
\end{tabular}

agnostic imaging has improved, which leads to detection of even small metastases, e.g., of the brain. Until now, up to $30 \%$ of patients with metastatic breast cancer develop brain metastases [4], which is particularly challenging for patients and therapists for two reasons. Firstly, patients with brain metastases, especially with hormone receptorpositive disease, have an unfavorable prognosis [5]. Moreover, brain metastases often cause severe neurological symptoms and cognitive and motor impairments and therefore significantly impact quality of life [3]. Currently, standard of care treatment includes neurosurgery and stereotactic and whole brain radiation, which is potentially associated with neurocognitive deficits. Because patients with breast cancer brain metastases are frequently excluded from clinical trials, there is little evidence of specific effectiveness of systemic therapies in this situation.

Here, we present the case of a woman with hormone receptor-positive, HER2-negative breast cancer with loss of sight and neurological symptoms due to orbital and cerebral metastases that improved clinically within 4 weeks of systemic treatment and vanished in the MRI scan 4 months after starting therapy with the CDK4/6 inhibitor ribociclib and the aromatase inhibitor anastrozole.

\section{Case Report}

Our patient was first diagnosed with early-stage breast cancer of the left breast at the age of 59 in 2009. Breast-conserving surgery and sentinel node biopsy was performed, and the postoperative TNM classification was pT1c pN0 (0/1) (sn) L0 V0 cM0 G2 R0. The tumor was estrogen receptor positive $(>90 \%)$, progesterone receptor positive (>90\%), and HER2 negative. The patient received adjuvant treatment with whole breast irradiation and adjuvant endocrine therapy with letrozole for 4 years.

In October 2017, the patient presented at the general practitioner with dyspnea and cough. A CT scan of the chest showed multiple suspicious masses in the lung, liver, and lymph nodes. As a result, the patient was referred to the breast cancer department, where staging was completed with an additional CT scan of the abdomen and a bone scan. Multiple suspicious masses were found in the liver with a size of up to $53 \times 45 \mathrm{~mm}$. In addition, bone metastases were diagnosed. A blood draw showed liver enzymes to be normal; body plethysmography revealed decompensated alkalosis, hypoxemia, mild obstruction, and no restriction.
A few days later, our patient noticed that her vision deteriorated and was referred to an ophthalmologist. The examination revealed a non-pigmented chorioretinal tumor, most likely matching a breast cancer metastasis, causing a serous amotio. The MRI scan performed showed at least five brain metastases as well as an intraorbital metastasis with thickening of the retina matching the ophthalmologist report and a bone metastasis of the skull.

To further evaluate tumor biology, we had a CT-targeted biopsy taken from the lung, which histologically confirmed a breast cancer metastasis of an estrogen receptor-positive (90\%), progesterone receptor-positive (20\%), HER2-negative (1+) tumor with a Ki-67 of $15 \%$.

We discussed the results with the patient and primarily recommended chemotherapy with paclitaxel in combination with bevacizumab considering the extensive tumor burden in liver, lung, and brain. Alternatively, an endocrine-based therapy with an aromatase inhibitor in combination with a CDK4/6 inhibitor was discussed.

As the patient refused the initially recommended whole brain radiation as well as any chemotherapy, treatment with anastrozole and ribociclib was started. We advised a second opinion for evaluation of stereotactic radiosurgery of the brain. By the time the patient had the appointment for second opinion, she had already taken the medication for 4 weeks. Prior symptoms were getting better, including the impaired vision, and dyspnea was markedly improved. The patient therefore opted against any form of local treatment.

The first follow-up staging after 2 months showed response to treatment resulting in a reduction in size of both pulmonary and hepatic metastases by more than $50 \%$. The head MRI showed stable disease of the brain metastases, but the retinal metastasis was reduced in size by more than $50 \%$. Our patient had become asymptomatic as she gained back normal vision and did not suffer from dyspnea anymore. Consequently, therapy with ribociclib and anastrozole was continued.

At the second staging 8 weeks later, all metastases, including the brain metastases, were reduced in size (Table 1); the intraorbital metastasis showed complete remission (Fig. 1). At that time, the patient herself described her quality of life as excellent.

Treatment was tolerated well apart from neutropenia, which led to a dose reduction of ribociclib from 600 to $400 \mathrm{mg} /$ day in May 2017. This dose reduction prevented further occurrence of neutropenia.

Another 3 months later, in the head MRI, radiologists described partly further shrinkage of cerebral metastases, partly stable disease. Clinically the patient still tolerated treatment well and did not show any symptoms. Thus, treatment was continued.

Nearly 9 months after starting treatment, staging with a CT scan of the chest and abdomen as well as a head MRI were carried out: apart from stable disease in the lung and brain (Fig. 2), significantly progressive disease in the liver $(8 \times 10 \mathrm{~cm})($ Fig. 3$)$ and the skull were observable. A liver biopsy was taken and confirmed tumor biology of a hormone receptor-positive (ER 80\%, PR 10\%) and HER2-negative breast cancer metastasis with a Ki-67 of $25 \%$. We counseled the patient on further treatment options and recommended a change in medication. But the patient wished to continue treatment with ribociclib and an aromatase inhibitor since she was mostly afraid of her brain metastases, which had remained stable throughout treatment. Due to worsening of her general condition she stopped taking ribociclib and continued anastrozole alone. The next staging after 2 months showed progressive disease in all metastatic sites apart from the brain. Transarterial chemoembolization of the liver metastases was done and chemotherapy with paclitaxel was started. Three cycles of chemotherapy were administered and showed good clinical response with a partial remission in liver and lung and stable disease in the brain. 
Fig. 1. Head MRI showing the intraorbital metastasis leading to amaurosis at the beginning of treatment with ribociclib and anastrozole (left) and its complete remission 8.5 months later (right).
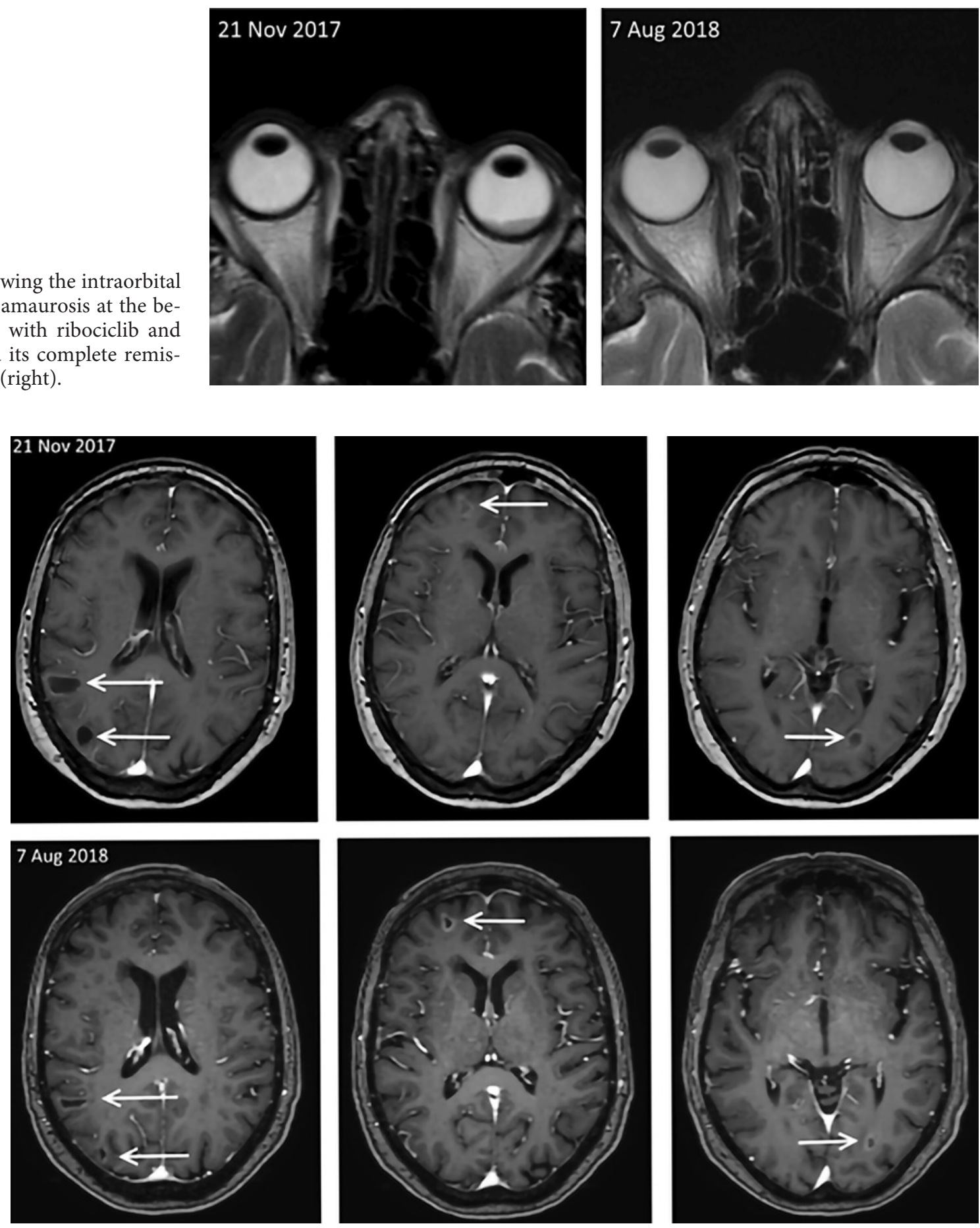

Fig. 2. Parenchymal brain metastases were all shown to be reduced in size in the staging MRI 3.5 months of initiating treatment with ribociclib and anastrozole (March 2018). This response remained stable until the last MRI 8.5 months after starting treatment.

\section{Discussion/Conclusion}

Breast cancer is the second most frequent cause of brain metastases. Trials have evaluated treatment strategies like surgery, radiation therapy (whole brain vs. stereotactic), and systemic treatment $[3,6-8]$.
To allow patients with metastatic breast cancer a relatively good quality of life, side effects of treatment options need to be considered and discussed with the patient. In hormone receptor-positive, HER2-negative metastatic breast cancer, first-line treatment should be based on endocrine therapy rather than chemotherapy unless there is 

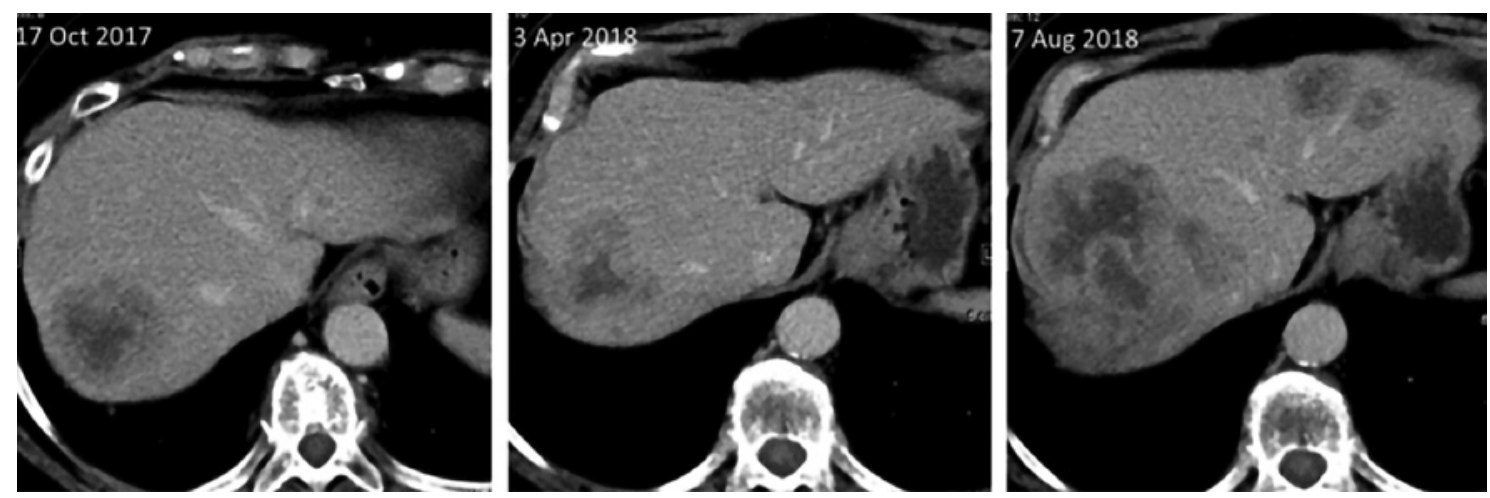

Fig. 3. Response to treatment of the liver metastases 5.5 months after initiating treatment with ribociclib and anastrozole. Another 4 months later, staging showed discordant response to treatment with progressive disease in the liver and stable disease of other metastatic sites.

impending organ failure and need for fast remission [911]. Several trials have shown that adding a CDK4/6 inhibitor to endocrine treatment improves progressionfree survival and overall survival in metastatic breast cancer patients with comparable health-related quality of life [12-18]. None of the trials with ribociclib excluded patients with cerebral metastases, whereas in the PALOMA trials with palbociclib, patients with known uncontrolled or symptomatic cerebral metastases were excluded. However, details about the number of patients with brain metastases and efficacy of the combined treatment with a CDK4/6 inhibitor and endocrine therapy in the ribociclib trials are lacking.

Pharmacokinetic analysis of ribociclib revealed $70 \%$ binding to human plasma protein with a 1:1 distribution between red blood cells and plasma. Ribociclib itself is the clinically effective agent, whereas its metabolites do not contribute much to the effectiveness. Our case shows clinical evidence that an endocrine combination therapy with ribociclib is effective in breast cancer brain metastases, which is also supported by preclinical data about the blood-brain barrier permeability of ribociclib and abemaciclib $[19,20]$. Other groups found poor drug delivery to the central nervous system [21]. Anastrozole has been shown to have limited effect in the central nervous system, too [22].

Due to the preclinical data in a phase II trial, patients with brain metastases from hormone receptor-positive breast cancer, NSCLC, or melanoma were treated with abemaciclib either alone or in combination with endocrine therapy. Out of 4 patients with brain metastases, one patient showed complete response of the parenchymal tumor; median overall survival in the heavily pretreated group was 8.4 months [23]. Median overall survival in patients with brain metastases from breast cancer is 3.5 months, with only $20 \%$ of the patients living longer than 1 year [24].
In conclusion, our case shows that in a patient with breast cancer brain metastases, a combination therapy of ribociclib and an aromatase inhibitor can lead to a rapid and durable response with clinical complete remission, even without initial brain surgery or radiation treatment. Therefore, systemic treatment with a CDK4/6 inhibitor and endocrine therapy can be considered in breast cancer brain metastases, especially if other treatment options are contraindicated or denied due to patient's preference. Further studies of CDK4/6 inhibitors in larger cohorts with breast cancer brain metastases are warranted.

\section{Statement of Ethics}

The patient consented to the publication. The published research complies with the guidelines for human studies and was conducted ethically in accordance with the World Medical Association Declaration of Helsinki.

\section{Disclosure Statement}

Joke Tio reports personal fees from Astra Zeneca, Daiichi Sankyo, Genomic Health, Eisai, Lilly, Novartis, Pfizer, Puma, Roche, Teva. Marie-Kristin von Wahlde: no conflicts of interest. Christoph Schülke: no conflicts of interest. Isabel Radke reports personal fees from Amgen, Astra Zeneca, Celgene, Novartis, Pfizer, Roche, Teva.

\section{Author Contributions}

All authors substantially contributed to the conception, data acquisition, and interpretation of the report. Marie-Kristin von Wahlde, Joke Tio, and Isabel Radke drafted the work, Christoph Schülke revised it critically. All authors finally approved the version to be published and agreed to be accountable for all aspects of the work ensuring that questions related to the accuracy or integrity of any part of the work are appropriately investigated and resolved. 


\section{References}

1 Global Burden of Disease Cancer Collaboration, Fitzmaurice C, Allen C, Barber RM, Barregard L, Bhutta ZA, et al. Global, Regional, and National Cancer Incidence, Mortality, Years of Life Lost, Years Lived With Disability, and Disability-Adjusted Life-years for 32 Cancer Groups, 1990 to 2015: A Systematic Analysis for the Global Burden of Disease Study. JAMA Oncol. 2017 Apr 1;3(4):524-48.

2 Gerratana L, Fanotto V, Bonotto M, Bolzonello S, Minisini AM, Fasola G, et al. Pattern of metastasis and outcome in patients with breast cancer. Clin Exp Metastasis. 2015 Feb; 32(2):125-33.

3 Gil-Gil MJ, Martinez-Garcia M, Sierra A, Conesa G, Del Barco S, González-Jimenez S, et al. Breast cancer brain metastases: a review of the literature and a current multidisciplinary management guideline. Clin Transl Oncol. 2014 May;16(5):436-46.

4 Tabouret E, Chinot O, Metellus P, Tallet A, Viens P, Gonçalves A. Recent trends in epidemiology of brain metastases: an overview. Anticancer Res. 2012 Nov;32(11):4655-62.

5 Witzel I, Laakmann E, Weide R, Neunhöffer T, Park-Simon TJ, Schmidt M, et al. Treatment and outcomes of patients in the Brain Metastases in Breast Cancer Network Registry. Eur J Cancer. 2018 Oct;102:1-9.

6 Kondziolka D, Patel A, Lunsford LD, Kassam A, Flickinger JC. Stereotactic radiosurgery plus whole brain radiotherapy versus radiotherapy alone for patients with multiple brain metastases. Int J Radiat Oncol Biol Phys. 1999 Sep;45(2):427-34.

7 Aoyama H, Shirato H, Tago M, Nakagawa K, Toyoda T, Hatano K, et al. Stereotactic radiosurgery plus whole-brain radiation therapy vs stereotactic radiosurgery alone for treatment of brain metastases: a randomized controlled trial. JAMA. 2006 Jun;295(21):2483-91.

8 Patil CG, Pricola K, Sarmiento JM, Garg SK, Bryant A, Black KL. Whole brain radiation therapy (WBRT) alone versus WBRT and radiosurgery for the treatment of brain metastases. Cochrane Database Syst Rev. 2017 Sep; 9:CD006121.
9 Stockler M, Wilcken N, Ghersi D, Simes RJ. The management of advanced breast cancer: systemic reviews of randomised controlled trials regarding the use of cytotoxic chemotherapy and endocrine therapy. Woolloomooloo: NHMRC National Breast Cancer Centre; 1997.

10 Rugo HS, Li H, Gui X. Strategies and Progress of Endocrine Therapy for Patients with Metastatic Breast Cancer. Adv Exp Med Biol. 2017; 1026:403-18.

11 Rugo HS, Rumble RB, Macrae E, Barton DL, Connolly HK, Dickler MN, et al. Endocrine Therapy for Hormone Receptor-Positive Metastatic Breast Cancer: American Society of Clinical Oncology Guideline. J Clin Oncol. 2016 Sep;34(25):3069-103.

12 O'Shaughnessy J, Petrakova K, Sonke GS, Conte P, Arteaga CL, Cameron DA, et al. Ribociclib plus letrozole versus letrozole alone in patients with de novo HR+, HER2- advanced breast cancer in the randomized MONALEESA-2 trial. Breast Cancer Res Treat. 2018 Feb;168(1):127-34.

13 Tripathy D, Im SA, Colleoni M, Franke F, Bardia A, Harbeck N, et al. Ribociclib plus endocrine therapy for premenopausal women with hormone-receptor-positive, advanced breast cancer (MONALEESA-7): a randomised phase 3 trial. Lancet Oncol. 2018 Jul; 19(7):904-15.

14 Slamon DJ, Neven P, Chia S, Fasching PA, De Laurentiis M, Im SA, et al. Phase III Randomized Study of Ribociclib and Fulvestrant in Hormone Receptor-Positive, Human Epidermal Growth Factor Receptor 2-Negative Advanced Breast Cancer: MONALEESA-3. J Clin Oncol. 2018 Aug;36(24):2465-72.

15 Verma S, O’Shaughnessy J, Burris HA, Campone M, Alba E, Chandiwana D, et al. Healthrelated quality of life of postmenopausal women with hormone receptor-positive, human epidermal growth factor receptor 2-negative advanced breast cancer treated with ribociclib + letrozole: results from MONALEESA-2. Breast Cancer Res Treat. 2018 Aug; 170(3):535-45.

16 Turner NC, Ro J, André F, Loi S, Verma S, Iwata $\mathrm{H}$, et al.; PALOMA3 Study Group. Palbociclib in Hormone-Receptor-Positive Advanced Breast Cancer. N Engl J Med. 2015 Jul; 373(3):209-19.
17 Loibl S, Turner NC, Ro J, Cristofanilli M, Iwata $\mathrm{H}$, Im SA, et al. Palbociclib Combined with Fulvestrant in Premenopausal Women with Advanced Breast Cancer and Prior Progression on Endocrine Therapy: PALOMA-3 Results. Oncologist. 2017 Sep;22(9):1028-38.

18 Im SA, Lu YS, Bardia A, Harbeck N, Colleoni M, Franke F, et al. Overall Survival with Ribociclib plus Endocrine Therapy in Breast Cancer. N Engl J Med. 2019 Jul;381(4):307-16.

19 Tripathy D, Bardia A, Sellers WR. Ribociclib (LEE011): Mechanism of Action and Clinical Impact of This Selective Cyclin-Dependent Kinase 4/6 Inhibitor in Various Solid Tumors Clin Cancer Res. 2017 Jul;23(13):3251-62.

20 Raub TJ, Wishart GN, Kulanthaivel P, Staton BA, Ajamie RT, Sawada GA, et al. Brain Exposure of Two Selective Dual CDK4 and CDK6 Inhibitors and the Antitumor Activity of CDK4 and CDK6 Inhibition in Combination with Temozolomide in an Intracranial Glioblastoma Xenograft. Drug Metab Dispos. 2015 Sep;43(9):1360-71.

21 Yin L, Li H, Liu W, Yao Z, Cheng Z, Zhang H, et al. A highly potent CDK4/6 inhibitor was rationally designed to overcome blood brain barrier in gliobastoma therapy. Eur J Med Chem. 2018 Jan; 144:1-28.

22 Miyajima M, Kusuhara $\mathrm{H}$, Takahashi K, Takashima T, Hosoya T, Watanabe Y, et al. Investigation of the effect of active efflux at the blood-brain barrier on the distribution of nonsteroidal aromatase inhibitors in the central nervous system. J Pharm Sci. 2013 Sep; 102(9):3309-19.

23 Tolaney SM, Sahebjam S, Le Rhun E, Lin NU, Bear MM, Yang Z, et al. Abstract P1-19-01: A phase 2 study of abemaciclib in patients with leptomeningeal metastases secondary to HR+, HER2- breast cancer. Cancer Res. 2019 Feb 15;79(4 suppl):P1-19-01.

24 Morikawa A, Jordan L, Rozner R, Patil S, Boire A, Pentsova E, et al. Characteristics and Outcomes of Patients With Breast Cancer With Leptomeningeal Metastasis. Clin Breast Cancer. 2017 Feb;17(1):23-8. 